\title{
Emergency department care of women who were abused was driven by the prevailing practice pattern of efficient patient processing
}

Varcoe C. Abuse obscured: an ethnographic account of emergency nursing in relation to violence against women.
Can J Nurs Res 2001 Mar;32:95-115.

QUESTION: What is the relation between the social context of practice and the ways in which nurses respond to women who have been abused?

Design

Critical ethnography.

\section{Setting}

Emergency departments (EDs) of 2 urban hospitals in Canada.

\section{Participants}

30 healthcare providers (21 white, female nurses and 9 other providers including admitting clerks, social workers, physicians, and administrators) and 5 women who had been abused.

\section{Methods}

Data were collected through $>200$ hours of observation and interviews. A core sample of 12 nurses, who were observed extensively, were interviewed twice for about 2 hours. Other healthcare providers and the women who had been abused were interviewed once for 1-3 hours. Analysis of transcribed interviews and field notes used established critical ethnography approaches.

\section{Main findings}

In general, abuse was obscured by practices required to keep the ED running. Abuse went largely unrecognised. Most nurses did not think they saw much abuse and recognised it only when there was serious physical injury, which fit the pattern of emergency practice. Abuse was obscured through efficient patient processing. The unpredictability of the ED and the sense of scarce resources required rapid, efficient patient processing. Patients were stripped down to "manageable problems", using physiological labels such as "laceration" or "fracture", with known solutions, which were processed so that patients could be moved out of the ED as quickly as possible. Nurses felt unable to attend to patients' psychosocial needs in general, and to abuse in particular. Abuse was obscured through stereotypical thinking. Nurses associated abuse with direct physical trauma in poor or "racialised" people only. They anticipated violence primarily among poor and "racialised" people, rather than among white, middle class, wealthy people.

When abuse was recognised, healthcare providers responded in 3 overlapping patterns: (1) Doing nothing for the undeserving victim. Recognising but not pursuing cues of abuse, only dealing with obvious physical injuries, and shifting responsibility to other providers or family members served the dominant practice of efficient patient processing. Doing nothing was justified by viewing the woman as undeserving of care because of perceived alcohol abuse, misuse of ED services, and failure to make changes to improve her life. (2) Influencing choices for the deserving victim. Nurses sometimes intervened with women whom they judged to be deserving of care, on the basis of social status and severity of physical injury. In such instances, providers guided women towards the "best" choices, which were largely limited to disclosing abuse, calling the police, and leaving the relationship. Women could be served efficiently if they accepted the choices offered; those who were unwilling to be influenced often came to be viewed as less deserving, which justified doing nothing further. (3) Offering choices to women with agency. When women were characterised as having personal agency, nurses tended to offer rather than influence choices. Offering choices required healthcare providers to give up control and comprised strategies such as listening and respecting choices. Offering choices sometimes ran counter to the pattern of practice because it required emotional and time commitments and thus reduced efficiency.

\section{Conclusions}

The responses of emergency department nurses to women who had been abused were influenced by the predominant practice pattern of efficient patient processing. This practice pattern and stereotypical thinking led to a focus on physical problems and obscured abuse. Perceptions of women's deservedness influenced the type of care provided, which ranged from doing nothing to offering choices.

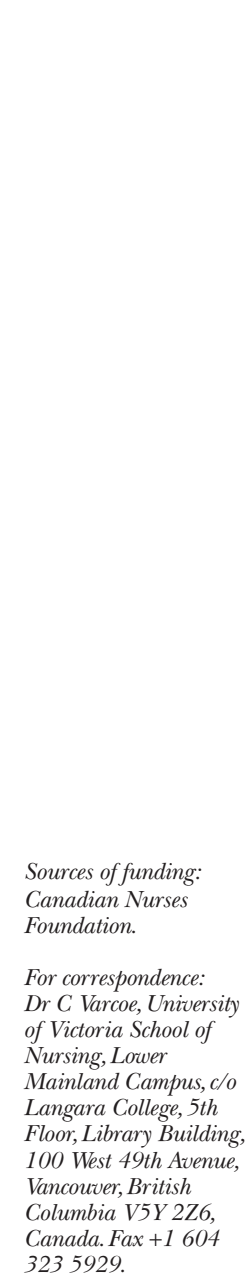

\section{COMMENTARY}

The issue of violence against women is an important area of research and deserves serious and focused attention. The study by Varcoe includes a current review of the literature related to violence against women. Varcoe builds on the literature and extends our knowledge by providing insight into nurses' experiences of working with this phenomenon in the ED. The nurses who were interviewed revealed the complexities of their work, how patient care was constrained by the larger system in which they work, and the frustrations they face in not being able to provide the care they believe is "best".

The method used, critical ethnography, is appropriate, allowing the researcher to obtain the perceptions of nurses, while at the same time raising nurses' own awareness of their clinical work. Through the interviews, nurses were able to reflect on and critique their own practices. There truly is a social action component of critical ethnography, in that nurses may become more motivated to seek some kind of constructive change within their job or occupational settings.

Important factors that influenced the nurses' work were revealed. One particular aspect of the nurses' work that was highlighted was the stereotypical judgments that the nurses often made about women on the basis of race, ethnicity, or social status. In addition, the tendency to emphasise physical symptoms because they allowed "rapid processing of patients" was addressed. These aspects allow a social analysis and critique of nursing in relation to working with abused women.

Varcoe has done an excellent job of uncovering the complexities of the nurse's role in working with women who have been abused, as well as the social contextual constraints that influence this role. In addition, she has provided an excellent example of critical ethnography that is useful in uncovering such complex information.

Ellen Olshansky, RNC, DNSc Professor and Associate Dean Duquesne University School of Nursing Pittsburgh, Pennsylvania, USA 